\title{
The Danish Bladder Cancer Database
}

This article was published in the following Dove Press journal:

Clinical Epidemiology

25 October 2016

Number of times this article has been viewed

\section{Erik Hansen ${ }^{1-3}$ \\ Heidi Larsson ${ }^{4}$ \\ Mette Nørgaard ${ }^{4}$ \\ Peter Thind ${ }^{3,5}$ \\ Jørgen Bjerggaard Jensen ${ }^{1-3}$}

'Department of Urology, Hospital of West Jutland-Holstebro, Holstebro, ${ }^{2}$ Department of Urology, Aarhus University Hospital, Aarhus, ${ }^{3}$ The Danish Bladder Cancer Database Group, ${ }^{4}$ Department of Clinical Epidemiology, Aarhus University Hospital, Aarhus, ${ }^{5}$ Department of Urology, Copenhagen University Hospital, Copenhagen, Denmark
Correspondence: Erik Hansen Department of Urology, Aarhus University Hospital, Palle Juul-Jensen Blvd 99, 8200 Aarhus N, Denmark Tel +45 61655577

Email erik.hansen@dadlnet.dk
Aim of database: The aim of the Danish Bladder Cancer Database (DaBlaCa-data) is to monitor the treatment of all patients diagnosed with invasive bladder cancer (BC) in Denmark.

Study population: All patients diagnosed with BC in Denmark from 2012 onward were included in the study. Results presented in this paper are predominantly from the 2013 population.

Main variables: In 2013, 970 patients were diagnosed with BC in Denmark and were included in a preliminary report from the database. A total of $458(47 \%)$ patients were diagnosed with non-muscle-invasive BC (non-MIBC) and 512 (53\%) were diagnosed with muscle-invasive BC (MIBC). A total of 300 (31\%) patients underwent cystectomy. Among the 135 patients diagnosed with MIBC, who were 75 years of age or younger, 67 (50\%) received neoadjuvent chemotherapy prior to cystectomy. In 2013, a total of 147 patients were treated with curativeintended radiation therapy.

Descriptive data: One-year mortality was 28\% (95\% confidence interval [CI]: 15-21). Oneyear cancer-specific mortality was 25\% (95\% CI: 22-27\%). One-year mortality after cystectomy was $14 \%$ (95\% CI: 10-18). Ninety-day mortality after cystectomy was 3\% (95\% CI: $1-5)$ in 2013. One-year mortality following curative-intended radiation therapy was $32 \%$ (95\% CI: 24-39) and 1-year cancer-specific mortality was 23\% (95\% CI: 16-31) in 2013.

Conclusion: This preliminary DaBlaCa-data report showed that the treatment of MIBC in Denmark overall meet high international academic standards. The database is able to identify Danish BC patients and monitor treatment and mortality. In the future, DaBlaCa-data will be a valuable data source and expansive observational studies on $\mathrm{BC}$ will be available.

Keywords: bladder cancer, cystectomy, neoadjuvant chemotherapy, curative-intended radiation therapy

\section{Aim of database}

Patients diagnosed with invasive bladder cancer $(\mathrm{BC})$ in Denmark are treated according to the national guidelines at one of the five uro-oncological centers. The aim of the Danish Bladder Cancer Database (DaBlaCa-data) is to monitor treatment and general survival of patients diagnosed with BC in Denmark. The goal of the database is to provide standards of the highest level of clinical evidence based on expert knowledge in the area.

To guide the selection of quality indicators suitable for monitoring treatment and outcome of BC in Denmark, a report reviewing the existing literature on the treatment of BC was made. ${ }^{1}$ In 2015, DaBlaCa-data consisted of three process indicators and ten outcome indicators concerning the diagnostic and treatment quality of patients diagnosed with BC in Denmark. Indicators may change over the years as the database evolves. Patients with noninvasive tumors (urothelial papillomas or carcinoma 
in situ) are not included in the database. To avoid extensive manual registration of data, the database is largely based on secondary data from the Danish National Registry of Patients (DNRP) and the Danish National Pathology Register (DNPR). ${ }^{2,3}$ Consequently, extensive validation of the data has been done to secure that the algorithms used to identify patients diagnosed with BC (stage T1-T4) and the treatment provided for these patients had sufficient quality. Only variables regarding the cause of death, used for estimating cancer-specific mortality, and use of neoadjuvant chemotherapy are registered manually. Patients are identified in the database by their personal security number, making it possible to link DaBlaCa-data to other Danish registries if so desired with a purpose of research or monitoring the quality of treatment.

\section{Study population}

All patients diagnosed with BC in Denmark are included in the database. Inclusion is based on the data generated by the algorithms, which subtracts patients from the DNRP and the DNPR on a regular basis from all hospitals and private clinics in Denmark.

In 2013, 970 patients were referred or allocated to one of the five uro-oncological treatment centers treating $\mathrm{BC}$ in Denmark and were included in the database. Of the diagnosed patients, $26 \%$ were women. The median age was 74 years for women and 73 years for men.

\section{Main variables}

In 2013, a total of 970 patients were diagnosed with $\mathrm{BC}$ in Denmark. Among them, 512 (53\%) were diagnosed with muscle-invasive $\mathrm{BC}$ (MIBC) and 458 were diagnosed with non-muscle-invasive $\mathrm{BC}$ (non-MIBC).

\section{Survival}

Of the 970 BC patients included in 2013, 698 were still alive 1 year after diagnosis, corresponding to a 1 -year mortality rate of $28 \%$ (95\% confidence interval [CI]: $25-31)$. The national standard had been set to $35 \%$ prior to evaluation based on a literature review. ${ }^{4}$ All five treatment centers met the standard.

In 2013, cancer-specific mortality 1 year after BC diagnosis was 25\% (95\% CI: 22-27). Of the 970 patients, 226 had died because of BC within 1 year after diagnosis, whereas for 54 cases $(6 \%)$, registration regarding the cause of death was missing. Therefore, cancer-specific death may in reality be higher than $25 \%$ but not higher than $31 \%$. The national standard was set at $15 \%$ or less. ${ }^{5}$ Only one of the five treatment centers met the standard (Table 1).

A total of 300 patients, diagnosed in 2013, underwent cystectomy due to BC. At cutoff date (April 15, 2015), last date for data to be included in the preliminary report, 1 -year follow-up after cystectomy was available for 270 patients, whereas data were missing for two patients. Therefore, 268 patients were included in this variable. Of them, 230 were still alive 1 year after BC diagnosis, corresponding to a 1-year mortality of $24 \%$ after cystectomy due to BC (95\% CI: 21-28) (Table 2). The standard was set at $30 \%$ as the highest. ${ }^{6}$ All treatment centers met the standard.

In 2013, 1-year cancer-specific mortality after cystectomy due to invasive $\mathrm{BC}$ was $7 \%(95 \% \mathrm{CI}$ : 5-11). A total of 19 patients were registered with $\mathrm{BC}$ as the cause of death, while 13 patients had missing data regarding the cause of death. The national standard was set at $12 \%$ or lower. ${ }^{7}$ All five centers met the standard.

\section{Fraction undergoing neoadjuvant treatment}

In all, 135 patients younger than 75 years were diagnosed with MIBC (stage T2-T4) and underwent cystectomy. Among them, 65 (48\%) received neoadjuvant chemotherapy prior to cystectomy. The national standard was set at $25 \%$ or higher. ${ }^{6}$ All treatment centers met the standard.

Table I One-year cancer-specific mortality after invasive bladder cancer diagnosis on a national level and per treatment center

\begin{tabular}{|c|c|c|c|c|c|c|c|}
\hline \multirow[t]{3}{*}{ Center } & & & \multirow{2}{*}{$\begin{array}{l}\text { Missing } \\
\text { information }\end{array}$} & \multicolumn{2}{|c|}{ Year } & & \\
\hline & \multicolumn{2}{|c|}{ Per diagnose year } & & 2013 & & \multicolumn{2}{|c|}{2012} \\
\hline & Standard met & Dead/total & n (\%) & $\%$ & $95 \% \mathrm{Cl}$ & $\%$ & $95 \% \mathrm{Cl}$ \\
\hline National & No & $226 / 921$ & $54(6)$ & 25 & $22-27$ & 24 & $21-27$ \\
\hline Herlev Hospital & No & $59 / 236$ & $10(4)$ & 25 & $20-31$ & 28 & $23-35$ \\
\hline Copenhagen University Hospital & No & $37 / 170$ & $12(7)$ & 22 & $16-29$ & 18 & $13-25$ \\
\hline Odense University Hospital & No & $58 / 198$ & $12(6)$ & 29 & $23-36$ & 23 & $17-29$ \\
\hline Aarhus University Hospital & No & $55 / 207$ & $17(8)$ & 27 & $21-33$ & 27 & $21-34$ \\
\hline Aalborg University Hospital & Yes & $17 / 110$ & $3(3)$ & 15 & $9-24$ & 21 & $14-30$ \\
\hline
\end{tabular}

Note: The national standard was set at $15 \%$ or less.

Abbreviation: $\mathrm{Cl}$, confidence interval. 
Table 2 One-year survival after cystectomy on a national level and per treatment center

\begin{tabular}{|c|c|c|c|c|c|c|c|}
\hline \multirow[t]{3}{*}{ Center } & \multicolumn{7}{|c|}{ Year } \\
\hline & \multicolumn{2}{|c|}{ Per diagnose year } & \multirow{2}{*}{$\begin{array}{l}\text { Missing } \\
\text { information }\end{array}$} & \multicolumn{2}{|c|}{2013} & \multicolumn{2}{|c|}{2012} \\
\hline & Standard met & Alive/total & & $\%$ & $95 \% \mathrm{Cl}$ & $\%$ & $95 \% \mathrm{Cl}$ \\
\hline National & Yes & $230 / 268$ & $2(1)$ & 86 & $81-90$ & 87 & $82-90$ \\
\hline Herlev Hospital & Yes & $44 / 47$ & $0(0)$ & 94 & $82-99$ & 96 & $85-99$ \\
\hline Copenhagen University Hospital & Yes & $57 / 69$ & $\mathrm{I}(\mathrm{I})$ & 83 & $72-91$ & 83 & $7|-9|$ \\
\hline Odense University Hospital & Yes & $38 / 45$ & $0(0)$ & 84 & $71-94$ & 86 & $76-93$ \\
\hline Aarhus University Hospital & Yes & $74 / 88$ & I (I) & 84 & $75-91$ & 83 & $73-90$ \\
\hline Aalborg University Hospital & Yes & $17 / 19$ & $0(0)$ & 89 & $67-99$ & 95 & $76-100$ \\
\hline
\end{tabular}

Note: The national standard was at least $70 \%$.

Abbreviation: $\mathrm{Cl}$, confidence interval.

\section{Fraction undergoing cystectomy}

In 2013, a total of 512 patients were diagnosed with MIBC (stage T2-T4) in Denmark. Among them, 166 (32\%) were cystectomized within 6 months. We found a variation of $23 \%$ $40 \%$ in this proportion among the five centers. The national standard for this variable is still undetermined.

\section{Post-cystectomy mortality}

The 90-day mortality in patients undergoing cystectomy due to invasive BC in Denmark was found to be 3\% (95\% CI: $1-5)$ in 2013 . The national standard was set at $7 \%$ or below and was met by all five centers. ${ }^{8}$

\section{Postsurgical admission after cystectomy}

For patients undergoing cystectomy due to BC in 2013, the median postsurgical admission including primary length of stay and readmissions after cystectomy during the first 90 days was 11 days (interquartile range [IQR]: 8-20 days). All admissions among the patients during the first 90 days were included, no matter the cause and the department. The national standard was set at a median admission of no more than 20 days. ${ }^{9}, 10$ All five centers met this standard.

\section{Oncological treatment}

In 2013, 147 patients diagnosed with BC received their first radiation therapy with curative intent. Of these, 99 were still alive 1 year after the date of first radiotherapy. One patient had undetermined status and was not included. Thus, 1-year mortality after curative radiation therapy due to BC was 32\% (95\% CI: 24-39). The national standard was set at $40 \%$ or lower. ${ }^{11}$ Two of the five centers did not meet the standard (1-year mortality of $42 \%$ and $43 \%$, respectively). The other three centers met the standard $(27 \%, 32 \%$, and $31 \%$, respectively).

Among the 147 patients receiving curative-intended radiation therapy in 2013, cancer-specific mortality was $23 \%$. Thirty-three patients were registered with $\mathrm{BC}$ as the cause of death. Three patients lacked information regarding the cause of death. The national standard was set at $18 \%$ or less. ${ }^{12}$ Only one of the five centers met the standard (11\%) (Table 3).

\section{Follow-up}

By April 15, 2015, patients registered in DaBlaCa-data are followed for only 2 and 3 years (2012 and 2013). The follow-up is continuous for each patient and ends at death of that patient. The data presented in this paper are based on

Table 3 One-year cancer-specific mortality after first curative-intended radiation therapy due to MIBC on a national level and per treatment center

\begin{tabular}{|c|c|c|c|c|c|c|c|}
\hline \multirow[t]{3}{*}{ Center } & & & & \multicolumn{4}{|c|}{ Year } \\
\hline & \multicolumn{2}{|c|}{ Per diagnose year } & \multirow{2}{*}{$\begin{array}{l}\text { Missing } \\
\text { information } \\
\mathbf{n}(\%)\end{array}$} & \multicolumn{2}{|c|}{2013} & \multicolumn{2}{|c|}{2012} \\
\hline & Standard met & Dead/total & & $\%$ & $95 \% \mathrm{Cl}$ & $\%$ & $95 \% \mathrm{Cl}$ \\
\hline National & No & $33 / 144$ & $3(2)$ & 23 & $|6-3|$ & 14 & $9-21$ \\
\hline Herlev Hospital & No & $9 / 44$ & $0(0)$ & 20 & $10-35$ & 3 & $0-14$ \\
\hline Copenhagen University Hospital & Yes & $2 / 19$ & $0(0)$ & 11 & $\mathrm{I}-33$ & 12 & $3-31$ \\
\hline Odense University Hospital & No & $|3 / 5|$ & $I(2)$ & 25 & $14-40$ & 19 & $8-33$ \\
\hline Aarhus University Hospital & No & $8 / 24$ & I (4) & 33 & $16-55$ & 26 & $9-51$ \\
\hline Aalborg University Hospital & No & $1 / 5$ & I (I7) & 20 & $\mathrm{I}-72$ & 18 & $2-52$ \\
\hline
\end{tabular}

Note: The national standard was set at $18 \%$ or less.

Abbreviations: MIBC, muscle-invasive bladder cancer; $\mathrm{Cl}$, confidence interval. 
a preliminary report including all patients diagnosed with BC in Denmark in the period between January 1, 2012 and December 31, 2013. DaBlaCa-data is an ongoing database enrolling all patients diagnosed with BC in Denmark from 2012 onward.

\section{Examples of research}

A validation report based on the validation of algorithms prior to the final establishment of DaBlaCa-data is in preparation. Further studies based on the results found in DaBlaCa-data are planned with a longer follow-up period.

\section{Conclusion}

DaBlaCa-data is able to identify Danish BC patients and monitor treatment and mortality. The preliminary DaBlaCa-data report showed comparable results between the five uro-oncological treatment centers responsible for the treatment of all patients diagnosed with $\mathrm{BC}$ in Denmark. Differences in mortality among the BC patients may reflect differences in the general mortality in different regions of Denmark.

The standard for cancer-specific mortality 1 year after $\mathrm{BC}$ diagnosis (15\%) is believed to be underestimated. Most studies of the existing literature focus on cancer-specific mortality in patients who had received treatment due to $\mathrm{BC}$. The DaBlaCa-data includes all patients with $\mathrm{BC}$; hence, $15 \%$ is an underestimate in this database, though $15 \%$ is the best estimate when reviewing the literature. The high 1-year survival rate after cystectomy could reflect the fact that all the five uro-oncological centers have high and uniform standards for selecting patients for cystectomy and high quality of the surgical procedure. The board members of DaBlaCa-data expect to see more variation in cancer-specific mortality over time, due to longer follow-up periods. Until now, the treatment strategy has differed concerning the trend to choose radiation therapy. All five centers strive toward more uniform treatment protocols in the future than in the past. The median length of stay in a hospital post-cystectomy was very low compared to the standard. This again may reflect the high and uniform surgical standard among the five uro-oncological centers in Denmark.

The variation seen among the centers regarding cancerspecific mortality after curative-intended radiotherapy may reflect differences in patient selection to radiotherapy. The fact that two of the five centers were slightly under the standard concerning 1-year survival after curative-intended radiotherapy is believed to be insignificant.

\section{Acknowledgments}

We deeply acknowledge Jakob Kristian Jakobsen for his reviewing report prior to the establishment of DaBlaCa-data; project nurse Anna Munk Nielsen for her huge validation work prior to the establishment of DaBlaCa-data; The Danish Cancer Society for their financial support; other board members in DaBlaCa-data: Niels Viggo Jensen, Anette Pedersen Pilt, Knud Fabrin; Elin Kallestrup, representative of the data controlling authority; and Anne Nakano Jensen, Quality Consultant MHSc.

DaBlaCa-data is an approved nationwide clinical quality database and is primarily financed by the Danish Regions. This paper was funded by the Program for Clinical Research Infrastructure (PROCRIN) established by the Lundbeck Foundation and the Novo Nordisk Foundation and administered by the Danish Regions. A grant of 450,000 Danish kroner was donated by The Danish Cancer Society to fund the validation process prior to the final establishment of DaBlaCa-data.

\section{Disclosure}

The authors report no conflicts of interest in this work.

\section{References}

1. Jakobsen JK. Dokumentalist Blarecancer, The Danish Clinical Registries. Available from: http://www.kcks-vest.dk/siteassets/de-kliniskedatabaser/dablaca/dokumentalistrapport-dablaca-data.pdf.

2. Andersen TF, Madsen M, Jørgensen J, Mellemkjoer L, Olsen JH. The Danish National Hospital Register. A valuable source of data for modern health sciences. Dan Med Bull. 1999;46(3):263-268.

3. Erichsen R, Lash TL, Hamilton-Dutoit SJ, Bjerregaard B, Vyberg M, Pedersen L. Existing data sources for clinical epidemiology: the Danish National Pathology Registry and Data Bank. Clin Epidemiol. 2010;2:51-56.

4. Lund L, Erichsen R, Norgaard M, Larsen EH, Borre M, Jacobsen J. Survival of invasive bladder cancer patients, 1998-2009: a central and northern Denmark population-based cohort study. Clin Epidemiol. 2011;3(Suppl 1):47-51.

5. Lughezzani G, Sun M, Shariat SF, et al. A population-based competingrisks analysis of the survival of patients treated with radical cystectomy for bladder cancer. Cancer. 2011;117(1):103-109.

6. Leow JJ, Martin-Doyle W, Rajagopal PS, et al. Adjuvant chemotherapy for invasive bladder cancer: a 2013 updated systemic review and metaanalysis of randomized trials. Eur Urol. 2014;66(1):42-54.

7. Otto W, May M, Fritsche HM, et al. Analysis of sex differences in cancer-specific survival and perioperative mortality following radical cystectomy: results of a large German multicenter study of nearly 2500 patients with urothelial carcinoma of the bladder. Gend Med. 2012;9(6):481-489.

8. Nielsen ME, Mallin K, Weaver MA, et al. The association of hospital volume with conditional 90-day mortality after cystectomy: an analysis of the National Cancer Database. BJU Int. 2014;114(1):46-55.

9. Arumainayagam N, McGrath J, Jefferson KP, Gillatt DA. Introduction of an enhanced recovery protocol for radical cystectomy. $B J U$ Int. 2008;101(6):698-701.

10. Berrum-Svennung I, Hedelin H, Holmang S. Costs of radical cystectomy. Scand J Urol Nephrol. 2005;39(1):36-41. 
11. Fokdal L, Hoyer M, von der Maase H. Treatment outcome and prognostic variables for local and survival in patients receiving radical radiotherapy for urinary bladder cancer. Acta Oncol. 2004;43(8):749-757.
12. Shelley MD, Barber J, Mason MD. Surgery versus radiotherapy for muscle invasive bladder cancer. Cochrane Database Syst Rev. 2001;3:CD002079.

\section{Publish your work in this journal}

Clinical Epidemiology is an international, peer-reviewed, open access, online journal focusing on disease and drug epidemiology, identification of risk factors and screening procedures to develop optimal preventative initiatives and programs. Specific topics include: diagnosis, prognosis, treatment, screening, prevention, risk factor modification,

Submit your manuscript here: http://www.dovepress.com/clinical-epidemiology-journal
Dovepress

systematic reviews, risk \& safety of medical interventions, epidemiology \& biostatistical methods, and evaluation of guidelines, translational medicine, health policies \& economic evaluations. The manuscript management system is completely online and includes a very quick and fair peer-review system, which is all easy to use. 